Conclusion: COMPASS-31score of more than 30 correlated with moderate to severe AND. Patients with severe AND develops significant hemodynamic fluctuations during surgery more so in prone.

\section{A038 Analgesic Efficacy of Ultrasound-Guided Quadratus Lumborum Block (QLB) for Anterior Iliac Crest Graft Site Pain in Patients Undergoing Cervical Corpectomy with Iliac Bone Grafting: A Retrospective Case Control Study Prasad Kanna, ${ }^{1}$ Rahul Pillai, ${ }^{1}$ Karen R. Lionel, ${ }^{1}$ Georgene Singh, ${ }^{1}$ Ramamani Mariappan ${ }^{1}$ ${ }^{1}$ Department of Anaesthesia, Christian Medical College (CMC), Vellore, Tamil Nadu, India}

Background: Pain after anterior iliac crest bone graft (AICBG) harvesting is severe enough to limit the postoperative ambulation and recovery and can also lead to chronic pain. Quadratus lumborum block (QLB) provides analgesia by depositing the local anesthetic in the thoracolumbar fascia which can spread from T9-L5 level and can thus provide effective analgesia.

Materials and Methods: All patients who underwent cervical corpectomy with AICBG from June 2017 to May 2019 were retrospectively studied. Necessary data were collected from computerized case records, inpatient hospital records and anesthesia records. QLB with catheter was placed in the beginning or at the end of surgery. Patients who did not receive QLB served as controls and patients who received QLB were taken as cases.

Results: A total of 25 patients were studied. The baseline demographics, intraoperative variables (opioid consumption and duration of anesthesia), and postoperative variables (time of ambulation and discharge time) between the groups were compared. All patients received 30 to $40 \mathrm{~mL}$ of either $0.2 \%$ ropivacaine or $0.25 \%$ bupivacaine for intraoperative analgesia. During the postoperative period, $30 \mathrm{~mL}$ of $0.2 \%$ ropivacaine or $0.2 \%$ bupivacaine was given $\mathrm{Q} 12 \mathrm{H}$ for 60 hours. There were no complications such as infection, hematoma or motor weakness noticed with QLB placement. Patients with QLB could be ambulated earlier compared with patients without block.

Conclusion: Administration of QLB decreased intraoperative opioid usage and helped in early ambulation and early discharge during the postoperative period.

\section{Acknowledgments}

We thank Dr. Srinivasan Swaminathan and Dr. Prasanna Bidkar from Department of Anesthesia at JIPMER, Puducherry for compiling the abstracts. 